\title{
Analytical determination of coronal parameters using the period ratio $P_{1} / 2 P_{2}$
}

\author{
M. P. McEwan, A. J. Díaz ${ }^{\star}$, and B. Roberts
}

\begin{abstract}
School of Mathematics and Statistics, University of St Andrews, St Andrews, Fife KY16 9SS, Scotland e-mail: bernie@mcs.st-and.ac.uk; toni.diaz@uib.es
\end{abstract}

Received 5 June 2007 / Accepted 30 November 2007

\begin{abstract}
Context. In transverse coronal loop oscillations, two periodicities have been measured simultaneously and are interpreted as the fundamental kink mode (with period $P_{1}$ ) and the first harmonic (with period $P_{2}$ ). Deviations of the period ratio $P_{1} / 2 P_{2}$ from unity provide information about the extent of longitudinal structuring within the loop.

Aims. Here we develop an analytical approximation that describes the shift in $P_{1} / 2 P_{2}$ in terms of the ratio $L / \Lambda_{\mathrm{c}}$ of the length $2 L$ of a coronal loop and the density scale height $\Lambda_{c}$.

Methods. We study the MHD wave equations in a low $\beta$ plasma using the thin tube approximation. Disturbances are described by a differential equation which may be solved for various equilibrium density profiles, obtaining dispersion relations in terms of Bessel functions. These dispersion relations may be used to obtain analytical approximations to the periods $P_{1}$ and $P_{2}$. We also present a variational approach to determining the period ratio and show how the WKB method may be used.

Results. Analytical approximations to the period ratio $P_{1} / 2 P_{2}$ are used to shed light on the magnitude of longitudinal structuring in a loop, leading to a determination of the density scale height. We apply our formula to the observations in Verwichte et al. (2004) and Van Doorsselaere et al. (2007), obtaining the coronal density scale height.

Conclusions. Our simple formula and approximate approaches highlight a useful analytical tool for coronal seismology. We demonstrate that $P_{1} / 2 P_{2}$ is linked to the density scale height, with no need for estimates of other external parameters. Given the accuracy of current observations, our formula provides a convenient means of determining density scale heights.
\end{abstract}

Key words. Sun: corona - Sun: oscillations - stars: coronae

\section{Introduction}

In the past techniques in coronal seismology have rested on the accuracy of relatively uncertain input parameters. For example, Roberts et al. (1984) argued that radio observations of coronal oscillations could be used in combination with calculations of the magnetohydrodynamic (MHD) modes of oscillation of a coronal flux tube to determine in situ physical conditions in the inhomogeneous corona. More recently, Nakariakov \& Ofman (2001) used the theory of the fast kink mode combined with TRACE observations to infer the magnetic field strength in a coronal loop, given reasonable estimates of plasma density. An extensive review of coronal seismology is given in Nakariakov \& Verwichte (2005).

It is important in coronal seismology to reduce the need for input parameters. Observations of higher harmonics, together with the more readily determined fundamental standing harmonic of a coronal loop, promises to shed light on the longitudinal structuring in a loop (Andries et al. 2005a; Andries et al. 2005b; Goossens et al. 2006; McEwan et al. 2006; Dymova \& Ruderman 2006a,b, 2007). A first observation was reported in Verwichte et al. (2004) where the fundamental kink mode and its first harmonic were observed simultaneously in an oscillating coronal loop. More recently, van Doorsselaere et al. (2007) also observed multiple harmonics oscillating in a coronal loop and

* Present address: Department de Física, Universitat de les Illes Balears, 07122 Palma de Mallorca, Spain. obtained measurements of the fundamental period and its first harmonic with a significantly improved accuracy. Observations of multiple oscillating harmonics have also been reported in De Moortel \& Brady (2007).

Andries et al. (2005b) argued that the ratio of the period of the fundamental mode of oscillation, $P_{1}$, to its first harmonic, of period $P_{2}$, contains information about the density stratification of the loop plasma. In a homogeneous string the ratio $P_{1} / 2 P_{2}$ is unity, but it deviates from this value when non-uniformity is considered; even in the standard loop model of Edwin \& Roberts (1983), density non-uniformity between the inside and outside of a cylindrical magnetic flux tube creates wave dispersion which, in turn, causes the period ratio $P_{1} / 2 P_{2}$ to fall below unity, though the effect is generally not as large as that due to longitudinal density variation (McEwan et al. 2006). McEwan et al. (2006) studied the ratio $P_{1} / 2 P_{2}$ in detail and identified the various effects that contribute towards the shift of this ratio from unity. Their conclusion is that longitudinal structuring of the plasma has the greatest impact on the shift of $P_{1} / 2 P_{2}$ from unity; consequently, the shift contains information about the longitudinal structuring of the plasma. Other applications involving the ratio of the fundamental mode of oscillation to higher harmonics are discussed in Erdélyi \& Verth (2007).

The thin tube approximation has been widely applied to the oscillations of photospheric flux tubes, stratified by gravity (see for example Ryutov \& Ryutova 1976; Roberts \& Webb 1978, 1979; Spruit 1981; Ferriz Mas \& Schüssler 1989; Stix 2004). 
Recently, Dymova \& Ruderman (2005, 2006a,b) showed its application to prominences and coronal flux tubes. Here, following Dymova \& Ruderman (2006a,b) and McEwan et al. (2006), we show that the thin tube approximation applied to coronal loops leads to an analytical determination of the effect of longitudinal structuring on the kink mode of oscillation. Of particular interest is that we are able to shed light on the nature of the period ratio $P_{1} / 2 P_{2}$, demonstrating its departure from unity as a result of longitudinal structuring in two specific models of that structuring. The period ratio is also determined by use of a variational principle and a WKB approach.

\section{Thin tube model}

Consider a zero- $\beta$ plasma embedded in an uniform magnetic field $\boldsymbol{B}_{0}=B_{0} \hat{z}$. The plasma density $\rho_{0}$ is considered to be structured along the magnetic field, so that $\rho_{0}=\rho_{0}(z)$; this arises naturally as a result of gravitational stratification, temperature stratification or of non-uniform heating of the loop. Then linear perturbations about such an equilibrium lead to the wave equations (Díaz et al. 2002; Díaz 2004)

$\frac{\partial^{2} p_{\mathrm{T}}}{\partial t^{2}}-c_{\mathrm{A}}^{2}(z) \nabla^{2} p_{\mathrm{T}}=0$,

$\rho_{0}(z)\left(\frac{\partial^{2}}{\partial t^{2}}-c_{\mathrm{A}}^{2}(z) \frac{\partial^{2}}{\partial z^{2}}\right) \boldsymbol{v}_{\perp}+\nabla_{\perp} \frac{\partial p_{\mathrm{T}}}{\partial t}=0$

where $p_{\mathrm{T}}$ denotes the perturbation in total pressure, $\boldsymbol{v}_{\perp}$ the perturbed velocity and the symbol $\perp$ stands for the components of the velocity and the gradient perpendicular to the equilibrium magnetic field. Here $c_{\mathrm{A}}(z)=B_{0} / \sqrt{\mu_{0} \rho_{0}(z)}$ denotes the Alfvén speed which varies along the field because of density variation; the profile for $\rho_{0}(z)$ is arbitrary. Equations (1) and (2) are valid inside the loop and also in the loop environment; the boundary conditions of continuous total pressure and velocity across the interface are required to connect the two regions. These equations were solved numerically in Donnelly et al. (2006) and Díaz et al. (2007).

Dymova \& Ruderman (2005, 2006a) have shown from Eqs. (1) and (2) that in the thin tube limit for non-axisymmetrical kink oscillations the radial velocity $v_{\mathrm{r}} \mathrm{e}^{\mathrm{i} \omega t}$ satisfies an equation of the form

$\frac{\mathrm{d}^{2} v_{\mathrm{r}}}{\mathrm{d} z^{2}}+\frac{\omega^{2}}{c_{\mathrm{k}}^{2}(z)} v_{\mathrm{r}}=0$,

where

$c_{\mathrm{k}}(z)=\left[\frac{2 B_{0}^{2}}{\mu_{0}\left(\rho_{\mathrm{i}}(z)+\rho_{\mathrm{e}}(z)\right)}\right]^{1 / 2}$

denotes the kink speed $c_{\mathrm{k}}$ in a tube with internal plasma density $\rho_{0}(z)=\rho_{\mathrm{i}}(z)$ and environment density $\rho_{\mathrm{e}}(z)$. Hereafter, the subscripts " $i$ " and "e" denote quantities internal or external to the loop, respectively. The thin tube Eq. (3) is amenable to an analytical treatment. Here we consider the solution of (3) for two profiles of $c_{\mathrm{k}}^{2}(z)$, an exponential profile and a linear profile. A number of other profiles have been explored by Dymova \& Ruderman (2006a,b) and Erdélyi \& Verth (2007). Notice that all the profiles discussed in this paper are symmetric around the $z=0$ plane, so we classify the solutions in even and odd modes, and we concentrate in the region with $0 \leq z \leq L$. Using symmetric profiles means also that at the apex point $z=0$ there is a cusp unless

$$
\left.\frac{\mathrm{d} \rho}{\mathrm{d} z}\right|_{z=0}=0
$$

which is not satisfied by some of the profiles discussed in the paper or in previous literature. However, the effects of this cusp are not particularly relevant for the period ratio, but it should be kept in mind.

\subsection{The exponential profile}

We consider the case of an exponential density profile. Suppose that the plasma densities $\rho_{\mathrm{i}}(z)$ inside and $\rho_{\mathrm{e}}(z)$ outside the loop are both exponentials with the same scale height $\Lambda_{c}$ :

$\rho_{\mathrm{i}}(z)=\rho_{\mathrm{i}}(0) \mathrm{e}^{z / \Lambda_{\mathrm{c}}}, \quad \rho_{\mathrm{e}}(z)=\rho_{\mathrm{e}}(0) \mathrm{e}^{z / \Lambda_{\mathrm{c}}} ;$

the density $\rho_{\mathrm{i}}(z)$ increases from $\rho_{\mathrm{i}}(0)$ at the loop apex $(z=0)$ to $\rho_{\mathrm{i}}(0) \mathrm{e}^{L / \Lambda_{\mathrm{c}}}$ at the loop footpoint $(z=L)$. For this choice of profile, the square of the kink speed $c_{\mathrm{k}}^{2}(z)=c_{\mathrm{k}}^{2}(0) \mathrm{e}^{-z / \Lambda_{\mathrm{c}}}$ is an exponentially decreasing function of $z$, and Eq. (3) becomes

$\frac{\mathrm{d}^{2} v_{\mathrm{r}}}{\mathrm{d} z^{2}}+\frac{\omega^{2}}{c_{\mathrm{k}}^{2}(0)} \mathrm{e}^{z / \Lambda_{\mathrm{c}}} v_{\mathrm{r}}=0$

Equation (7) may be solved in terms of the zeroth order Bessel functions $J_{0}$ and $Y_{0}$ (Abramowitz \& Stegun 1964), yielding

$v_{\mathrm{r}}(z)=A J_{0}\left(\lambda \mathrm{e}^{z / 2 \Lambda_{\mathrm{c}}}\right)+B Y_{0}\left(\lambda \mathrm{e}^{z / 2 \Lambda_{\mathrm{c}}}\right)$,

where $\lambda=2 \Lambda_{\mathrm{c}} \omega / c_{\mathrm{k}}(0)$.

Consider the standing modes of oscillation of a loop of length $2 L$. Line-tying at the photospheric/chromospheric base requires that $v_{\mathrm{r}}=0$ at $z=L$. Conditions at the loop apex $z=0$ determine the modes of oscillation. For even modes, the radial velocity $v_{\mathrm{r}}$ is symmetric about the loop apex so $\mathrm{d} v_{\mathrm{r}} / \mathrm{d} z=0$ at $z=0$; the oscillation has a maximum or minimum at the loop apex. The dispersion relation for the even modes follows from Eq. (8) and these boundary conditions:

$J_{0}^{\prime}(\lambda) Y_{0}\left(\lambda \mathrm{e}^{L / 2 \Lambda_{\mathrm{c}}}\right)-Y_{0}^{\prime}(\lambda) J_{0}\left(\lambda \mathrm{e}^{L / 2 \Lambda_{\mathrm{c}}}\right)=0$

Here a dash $\left({ }^{\prime}\right)$ denotes the derivative of a Bessel function: $J_{0}^{\prime}(\lambda)=\mathrm{d} J_{0} / \mathrm{d} z$ calculated at $z=\lambda$.

Similarly, the odd modes have a radial motion $v_{\mathrm{r}}$ that has a node at the loop apex $z=0$ as well as the loop base $z=L$, and these modes satisfy the dispersion relation

$J_{0}(\lambda) Y_{0}\left(\lambda \mathrm{e}^{L / 2 \Lambda_{\mathrm{c}}}\right)-Y_{0}(\lambda) J_{0}\left(\lambda \mathrm{e}^{L / 2 \Lambda_{\mathrm{c}}}\right)=0$

We consider approximate solutions of the dispersion relations (9) and (10). Since

$\lambda=\frac{2 \Lambda_{\mathrm{c}} \omega}{c_{\mathrm{k}}(0)}=2 \frac{\Lambda_{\mathrm{c}}}{L} \frac{\omega L}{c_{\mathrm{Ai}}}\left[\frac{1}{2}\left(1+\frac{c_{\mathrm{Ai}}^{2}}{c_{\mathrm{Ae}}^{2}}\right)\right]^{1 / 2}$,

the parameter $\lambda$ is large whenever $\omega \gg c_{\mathrm{k}}(0) / 2 \Lambda_{\mathrm{c}}$. Standing kink waves in a uniform loop of length $2 L$ result in periods $4 L / n c_{\mathrm{k}}$, with $n=1,2, \ldots$, giving $\lambda \gg 1$ whenever $L / \Lambda_{\mathrm{c}} \ll n \pi$; so we may expect $\lambda \gg 1$ in loops that are much shorter than $\pi \Lambda_{c}$. This is essentially the case of weak longitudinal stratification. 
Suppose, then, that $\lambda$ is large. We may then employ the expansions for Bessel functions of large arguments (Abramowitz \& Stegun 1964):

$$
\begin{aligned}
J_{0}(Z) \simeq & \sqrt{\frac{2}{\pi Z}}\left(\left(1-\frac{9}{128 Z^{2}}\right) \cos (Z-\pi / 4)\right. \\
& \left.+\frac{1}{8 Z} \sin (Z-\pi / 4)\right), \\
Y_{0}(Z) \simeq & \sqrt{\frac{2}{\pi Z}}\left(\left(1-\frac{9}{128 Z^{2}}\right) \sin (Z-\pi / 4)\right. \\
& \left.-\frac{1}{8 Z} \cos (Z-\pi / 4)\right), \\
J_{0}^{\prime}(Z) \simeq & \sqrt{\frac{2}{\pi Z}}(-\cos (Z-3 \pi / 4) \\
& \left.+\frac{3}{8 Z} \sin (Z-3 \pi / 4)\right),
\end{aligned}
$$

and

$$
\begin{aligned}
Y_{0}^{\prime}(Z) \simeq & \sqrt{\frac{2}{\pi Z}}(-\sin (Z-3 \pi / 4) \\
& \left.-\frac{3}{8 Z} \cos (Z-3 \pi / 4)\right) .
\end{aligned}
$$

Substituting these expansions into Eq. (9), we obtain for the even mode

$$
\begin{aligned}
\{1+ & \left.\frac{1}{128 \lambda^{2}}\left(15-\frac{6}{\mathrm{e}^{L / 2 \Lambda_{\mathrm{c}}}}-\frac{9}{\mathrm{e}^{L / \Lambda_{\mathrm{c}}}}\right)\right\} \\
& \times \cot \left[\lambda\left(\mathrm{e}^{L / 2 \Lambda_{\mathrm{c}}}-1\right)\right]+\frac{1}{8 \lambda} \frac{3 \mathrm{e}^{L / 2 \Lambda_{\mathrm{c}}}+1}{\mathrm{e}^{L / 2 \Lambda_{\mathrm{c}}}}=0,
\end{aligned}
$$

we have neglected terms of order $\lambda^{-3}$.

Writing $\lambda=(2 \Omega) /\left(L / \Lambda_{\mathrm{c}}\right)$ and $\Omega=\pi / 2+\left(L / \pi \Lambda_{\mathrm{c}}\right) \Omega_{1}+$ $\left(L / \pi \Lambda_{\mathrm{c}}\right)^{2} \Omega_{2}+\ldots$, we may obtain an equation approximating the first zero of Eq. (16), i.e. the fundamental period $P_{1}$ for $L / \Lambda_{\mathrm{c}} \ll \pi$ :

$$
\begin{array}{r}
\left(\frac{L}{\pi \Lambda_{\mathrm{c}}}\right)\left(\frac{1}{2}-\Omega_{1}-\frac{\pi^{2}}{8}\right)-\left(\frac{L}{\pi \Lambda_{\mathrm{c}}}\right)^{2}\left(\frac{\Omega_{1}}{\pi}+\frac{\pi}{16}\right. \\
\left.+\Omega_{2}+\frac{\pi \Omega_{1}}{4}+\frac{\pi^{3}}{48}\right)+O\left(\frac{L}{\pi \Lambda_{\mathrm{c}}}\right)^{3}=0 .
\end{array}
$$

This determines $\Omega_{1}$ and $\Omega_{2}$. Thus, for the even mode, $\Omega=\Omega_{\text {even }}$ where

$$
\begin{aligned}
\Omega_{\text {even }} \simeq \frac{\pi}{2}- & \left(\frac{\pi^{2}}{8}-\frac{1}{2}\right)\left(\frac{L}{\pi \Lambda_{\mathrm{c}}}\right) \\
& +\left(\frac{\pi^{3}}{96}-\frac{\pi}{16}-\frac{1}{2 \pi}\right)\left(\frac{L}{\pi \Lambda_{\mathrm{c}}}\right)^{2}+\ldots
\end{aligned}
$$

Equation (18) determines the period $P_{1}=2 \pi / \omega$ of the fundamental mode of the loop as a whole:

$$
P_{1}=\frac{P_{\mathrm{k}}}{1-\left(\frac{\pi}{4}-\frac{1}{\pi}\right)\left(\frac{L}{\pi \Lambda_{\mathrm{c}}}\right)+\left(\frac{\pi^{2}}{48}-\frac{1}{8}-\frac{1}{\pi^{2}}\right)\left(\frac{L}{\pi \Lambda_{\mathrm{c}}}\right)^{2}},
$$

where $P_{\mathrm{k}}$ is the fundamental period of a fast kink wave in a $u n i$ form loop $\left(\Lambda_{\mathrm{c}} \rightarrow \infty\right)$,

$$
P_{\mathrm{k}}=\frac{4 L}{c_{\mathrm{k}}(0)}=4 \frac{L}{c_{\mathrm{Ai}}}\left[\frac{1}{2}\left(1+\frac{c_{\mathrm{Ai}}^{2}}{c_{\mathrm{Ae}}^{2}}\right)\right]^{1 / 2} .
$$

In the limit of a uniform loop $\left(\Lambda_{\mathrm{c}} \rightarrow \infty\right)$, Eq. (19) reduces to $P_{1}=P_{\mathrm{k}}$.

In a similar way, expanding Eq. (10), we obtain for the odd mode

$$
\begin{aligned}
\{1- & \left.\frac{9}{128 \lambda^{2}}\left(1-\frac{2}{9 \mathrm{e}^{L / 2 \Lambda_{\mathrm{c}}}}+\frac{1}{\mathrm{e}^{L / \Lambda_{\mathrm{c}}}}\right)\right\} \\
& \times \tan \left[\lambda\left(\mathrm{e}^{L / 2 \Lambda_{\mathrm{c}}}-1\right)\right]+\frac{1}{8 \lambda} \frac{\mathrm{e}^{L / 2 \Lambda_{\mathrm{c}}}-1}{\mathrm{e}^{L / 2 \Lambda_{\mathrm{c}}}}=0,
\end{aligned}
$$

which, expanded as a series in $L / \pi \Lambda_{\mathrm{c}}$, yields

$x_{\text {odd }} \simeq \pi-\frac{\pi^{2}}{4}\left(\frac{L}{\pi \Lambda_{\mathrm{c}}}\right)+\left(\frac{\pi^{3}}{48}-\frac{\pi}{32}\right)\left(\frac{L}{\pi \Lambda_{\mathrm{c}}}\right)^{2}$.

This gives the approximation of $P_{2}$ to second order:

$$
P_{2}=\frac{P_{\mathrm{k}} / 2}{1-\frac{\pi}{4}\left(\frac{L}{\pi \Lambda_{\mathrm{c}}}\right)+\left(\frac{\pi^{2}}{48}-\frac{1}{32}\right)\left(\frac{L}{\pi \Lambda_{\mathrm{c}}}\right)^{2}} .
$$

Equation (23) gives the period of the first harmonic of the loop as a whole.

\subsection{The linear profile}

It is of interest to consider other profiles of $c_{\mathrm{k}}^{2}(z)$ in addition to the exponential case. Consider the case when the kink speed squared, $c_{\mathrm{k}}^{2}$, varies linearly with $z$ :

$c_{\mathrm{k}}^{2}(z)=c_{\mathrm{k}}^{2}(0)(1-\alpha z)$.

The corresponding density profiles are

$\rho_{\mathrm{i}}(z)=\frac{\rho_{\mathrm{i}}(0)}{(1-\alpha z)}, \quad \rho_{\mathrm{e}}(z)=\frac{\rho_{\mathrm{e}}(0)}{(1-\alpha z)}$.

The density $\rho_{\mathrm{i}}(z)$ increases from $\rho_{\mathrm{i}}(0)$ at the loop apex to $\rho_{\mathrm{i}}(0) /(1-\alpha L)$ at the loop base. The parameter $\alpha$ plays the same role as $\Lambda_{\mathrm{c}}^{-1}$; indeed, near the loop apex, the exponential profile gives

$c_{\mathrm{k}}^{2}(z)=c_{\mathrm{k}}^{2}(0) \mathrm{e}^{-z / \Lambda_{\mathrm{c}}} \simeq c_{\mathrm{k}}^{2}(0)\left(1-\frac{z}{\Lambda_{\mathrm{c}}}\right), \quad|z| \ll \Lambda_{\mathrm{c}}$.

For the profile (24), Eq. (3) becomes

$\frac{\mathrm{d}^{2} v_{\mathrm{r}}}{\mathrm{d} z^{2}}+\frac{\omega^{2}}{c_{\mathrm{k}}^{2}(0)(1-\alpha z)} v_{\mathrm{r}}=0$

which has solution

$$
\begin{aligned}
v_{\mathrm{r}}(z)= & (1-\alpha z)^{1 / 2}\left(A J_{1}\left(\lambda_{1}(1-\alpha z)^{1 / 2}\right)\right. \\
& \left.+B Y_{1}\left(\lambda_{1}(1-\alpha z)^{1 / 2}\right)\right),
\end{aligned}
$$

where $\lambda_{1}=2 \omega / c_{\mathrm{k}}(0) \alpha$.

The dispersion relation for the even kink modes of oscillation in a thin coronal loop of length $2 L$, with a linearly varying kink speed squared (embedded in an atmosphere also with a linearly varying kink speed squared) is

$J_{0}\left(\lambda_{1}\right) Y_{1}\left(\lambda_{1} b\right)-Y_{0}\left(\lambda_{1}\right) J_{1}\left(\lambda_{1} b\right)=0$ 
where

$b=(1-\alpha L)^{1 / 2}=c_{\mathrm{k}}(L) / c_{\mathrm{k}}(0)$

is the ratio of the kink speed at the base to that at the apex. The odd modes satisfy

$J_{1}\left(\lambda_{1}\right) Y_{1}\left(\lambda_{1} b\right)-Y_{1}\left(\lambda_{1}\right) J_{1}\left(\lambda_{1} b\right)=0$.

Again, we may obtain approximate solutions of these dispersion relations, much as in the exponential case. Following similar steps than those in Sect. 2.1, one can obtain the following expressions for the periods of the fundamental mode and its first harmonic:

$$
\begin{aligned}
& P_{1}=\frac{P_{\mathrm{k}}}{1-\left(\frac{\pi}{4}-\frac{1}{\pi}\right)\left(\frac{L}{\pi \Lambda_{\mathrm{c}}}\right)+\left(\frac{3}{8}-\frac{\pi^{2}}{16}-\frac{1}{\pi^{2}}\right)\left(\frac{L}{\pi \Lambda_{\mathrm{c}}}\right)^{2}}, \\
& P_{2}=\frac{P_{\mathrm{k}} / 2}{1-\frac{\pi}{4}\left(\frac{L}{\pi \Lambda_{\mathrm{c}}}\right)+\left(\frac{3}{32}-\frac{\pi^{2}}{16}\right)\left(\frac{L}{\pi \Lambda_{\mathrm{c}}}\right)^{2}},
\end{aligned}
$$

where again $P_{\mathrm{k}}$ is the fundamental period of a fast kink wave in a uniform loop. We can see that up to first order they are identical to their counterparts for the exponential profile, which might be expected, since the linear profile can be viewed as a first order approximation (Eq. (26)).

\subsection{A variational approach}

The thin tube equation, Eq. (3), is amenable to an approximate treatment, as well as the exact solutions for certain specific profiles. Multiplying Eq. (3) by $v_{\mathrm{r}}$ and integrating along the loop from the apex to the base gives

$$
\int_{0}^{L} v_{\mathrm{r}} \frac{\mathrm{d}^{2} v_{\mathrm{r}}}{\mathrm{d} z^{2}} \mathrm{~d} z+\omega^{2} \int_{0}^{L} v_{\mathrm{r}}^{2} \frac{1}{c_{\mathrm{k}}^{2}(z)} \mathrm{d} z=0 .
$$

The first integral arising here may be treated using integration by parts. The boundary conditions, $v_{\mathrm{r}}=0$ at the base $(z=L)$ and $v_{\mathrm{r}} \mathrm{d} v_{\mathrm{r}} / \mathrm{d} z=0$ at the loop apex, which apply for the even or odd modes, leads to the results that

$\omega^{2}=\frac{I}{J}$,

on use of integration by parts. Here

$I=\int_{0}^{L}\left(\frac{\mathrm{d} v_{\mathrm{r}}}{\mathrm{d} z}\right)^{2} \mathrm{~d} z, \quad J=\int_{0}^{L} \frac{1}{c_{\mathrm{k}}^{2}(z)} v_{\mathrm{r}}^{2} \mathrm{~d} z$.

Thus, evaluation of the integrals $I$ and $J$, given a specific profile for $c_{\mathrm{k}}^{2}(z)$ together with knowledge of the eigenfunction $v_{\mathrm{r}}(z)$, determines the frequency $\omega$. Equation (35) provides a variational formulation of our eigenvalue problem used in the Rayleigh-Ritz procedure (e.g. Morse \& Feshbach 1953). We can exploit this approach here for any specific profile $c_{\mathrm{k}}^{2}(z)$, by taking trial functions for $v_{\mathrm{r}}$ which satisfy the boundary conditions at the loop apex and base. For example, we may choose

$v_{\mathrm{r}}(z)= \begin{cases}\cos \left(\frac{\pi z}{2 L}\right), & \text { for } P_{1}, \\ \sin \left(\frac{\pi z}{L}\right), & \text { for } P_{2} .\end{cases}$

These trial functions are chosen to give the appropriate behaviour for $v_{\mathrm{r}}(z)$ that accords with the fundamental mode of period $P_{1}$ and its first harmonic of period $P_{2}$. Equation (35) determines the frequency $\omega$ (and so the periods $P_{1}$ and $P_{2}$ ) for any choice of profile $c_{\mathrm{k}}^{2}(z)$, by evaluating the integrals $I$ and $J$.

\subsection{WKB approximation}

The variational approach leading to Eq. (34) requires trial functions which may either be chosen for simplicity (as in Sect. 2.3) or by methods that lead to more accurate representations of the actual eigenfunctions. The WKB method provides a powerful and convenient means of generating such functions (Bender \& Orszag 1978).

We write Eq. (3) in the form

$\frac{\mathrm{d}^{2} v_{\mathrm{r}}}{\mathrm{d} s^{2}}+\mu^{2} f(s) v_{\mathrm{r}}=0$

where

$\mu=\frac{\omega L}{c_{\mathrm{k}}(0)}, \quad f(z)=\frac{c_{\mathrm{k}}^{2}(0)}{c_{\mathrm{k}}^{2}(z)}, \quad s=z / L$,

Notice that $f(0)=1$.

Considering $\mu \gg 1$, we expand $v_{\mathrm{r}}(s)$ as

$v_{\mathrm{r}}(s)=\mathrm{e}^{\mathrm{i}\left(\Phi_{0} \mu+\Phi_{1}+\Phi_{2} / \mu\right)}$,

with $\Phi_{0}(s), \Phi_{1}(s)$ and $\Phi_{2}(s)$ to be determined. Substituting Eq. (40) into (38) allows us to equate powers of $\mu$, obtaining a hierarchy of equations starting with:

$$
\begin{aligned}
-\Phi_{0}^{\prime 2}+f=0 & \Rightarrow \Phi_{0}(s)=\int_{0}^{s} \sqrt{f(r)} \mathrm{d} r \\
\mathrm{i} \Phi_{0}^{\prime \prime}-2 \Phi_{0}^{\prime} \Phi_{1}^{\prime}=0 & \Rightarrow \Phi_{1}(s)=\frac{\mathrm{i}}{4} \log (f(s)) \\
\mathrm{i} \Phi_{0}^{\prime \prime}-\Phi_{1}^{\prime 2}-2 \Phi_{0}^{\prime} \Phi_{2}^{\prime}=0 & \Rightarrow \\
\Phi_{2}(s) & =\int_{0}^{s} \frac{f^{\prime 2}(r)-4 f(r) f^{\prime \prime}(r)}{32(f(r))^{5 / 2}} \mathrm{~d} r .
\end{aligned}
$$

Both $\Phi_{0}$ and $\Phi_{2}$ are real functions of $f(s)$, so the solution to Eq. (38) may be written in the form

$$
\begin{array}{r}
v_{\mathrm{r}}(s)=\frac{1}{(f(s))^{1 / 4}}\left[A \cos \left(\Phi_{0} \mu+\Phi_{2} / \mu\right)\right. \\
\left.+B \sin \left(\Phi_{0} \mu+\Phi_{2} / \mu\right)\right] .
\end{array}
$$

The boundary conditions determine the eigenfrequencies. Firstly, for the odd modes we require $v_{\mathrm{r}}(0)=0$, so $A=0$; then the line-tying boundary condition $v_{\mathrm{r}}(z=L)=0$ leads to

$\mu_{2}=\frac{\pi \pm \sqrt{\pi^{2}-4 \Phi_{0}(1) \Phi_{2}(1)}}{2 \Phi_{0}(1)}$,

which determines $\mu=\mu_{2}$, the value of $\mu$ for the first harmonic choosing the positive sign. It turns out numerically that the negative root in Eq. (43) leads to a small $\mu$, incompatible with the assumption $\mu \gg 1$ in the WKB expansion Eq. (40).

Obtaining an explicit expression for the first eigenvalue $\mu=$ $\mu_{1}$ is more difficult. For the even modes, $v_{\mathrm{r}}^{\prime}(0)=0$; this gives a relation between $A$ and $B$ in Eq. (42). The line-tying boundary condition $v_{\mathrm{r}}(z=L)=0$ then implies a transcendental equation for $\mu_{1}$ :

$$
\tan \left[\Phi_{0}(1) \mu_{1}+\Phi_{2}(1) / \mu_{1}\right]=\frac{-32 \mu_{1}^{2}-5 f^{\prime}(0)+4 f^{\prime \prime}(0)}{8 \mu_{1} f^{\prime}(0)} .
$$

Equations (43) and (44) can be solved numerically, and then used it to obtain an approximation to the period ratio as

$$
\frac{P_{1}}{2 P_{2}}=\frac{\mu_{2}}{2 \mu_{1}} \text {. }
$$


Higher orders in the WKB approximation could be used if more accurate approximations are necessary.

Expressions (43) and (44) may be used to approximate the period ratio for the exponential and linear profiles discussed earlier, and the agreement they give is excellent. These formulae may also used to approximate the period for different density profiles, even if analytical or numerical solutions are difficult to obtain, needing only the integrals in Eq. (41) to be evaluated for $s=1$ and then the transcendental Eq. (44) to be solved to obtain an approximation for the period ratio.

\section{Discussion}

We have pointed out a number of ways in which the period ratio $P_{1} / 2 P_{2}$ may be determined, using either analytical solutions and their approximations for specific profiles of $c_{\mathrm{k}}^{2}(z)$ or by considering a variational or $\mathrm{WKB}$ formulation for these profiles or other choices. We now return to the exponential profile, with the aim of using our analytical results to deduce the scale $\Lambda_{c}$ of variation in $c_{\mathrm{k}}^{2}(z)$ from observations. Other profiles give similar results to the exponential case.

Consider then the periods $P_{1}$ and $P_{2}$ in a loop that is exponentially structured (with density profiles given by Eq. (6)). Equations (19) and (23) determine these periods in terms of $P_{\mathrm{k}}$, the period of a kink mode in a loop that has no longitudinal structuring; $P_{\mathrm{k}}$ depends upon the loop length $2 L$, the internal and external Alfvén speeds, and the internal and external densities. However, by combining $P_{1}$ and $P_{2}$ to form their ratio we may eliminate $P_{\mathrm{k}}$, obtaining a period ratio $P_{1} / 2 P_{2}$ which depends upon the ratio $L / \Lambda_{\mathrm{c}}$ of loop half length $L$ to density scale height $\Lambda_{\mathrm{c}}$ :

$$
\frac{P_{1}}{2 P_{2}}=1-\frac{1}{\pi^{2}} \frac{L}{\Lambda_{\mathrm{c}}}+\left(\frac{2}{\pi^{4}}-\frac{5}{32 \pi^{2}}\right)\left(\frac{L}{\Lambda_{\mathrm{c}}}\right)^{2} .
$$

It is interesting to note that the linear profile gives a very similar result to this, with the only change being that the coefficient 5/32 is replaced by $17 / 32$.

Figure 1 shows the variation of $P_{1} / 2 P_{2}$ with $L / \Lambda_{\mathrm{c}}$ as determined for the exponential profile; we show the approximate formula (46) together with the exact solution of the full dispersion relations (9) and (10). The agreement is excellent (and incidentally also agrees with a full numerical solution of Eqs. (1) and (2) carried out by Díaz et al. 2007).

The distance $\Lambda_{c}$ is a measure of the scale of longitudinal variation in the square of the kink speed, $c_{\mathrm{k}}^{2}$. In the exponential profile, $\Lambda_{\mathrm{c}}$ is the scale height of $c_{\mathrm{k}}^{2}$ at all points along the loop. The assumption of similar profiles in internal plasma density $\rho_{\mathrm{i}}(z)$ and external plasma density $\rho_{\mathrm{e}}(z)$ allows us to relate $\Lambda_{\mathrm{c}}$ to the density scale height. We can express these quantities in terms of the ratio $\rho_{\text {apex }} / \rho_{\text {base }}$ of internal density $\rho_{\text {apex }}=\rho_{\mathrm{i}}(z=0)$ at the loop apex to the density $\rho_{\text {base }}=\rho_{\mathrm{i}}(L)$ at the loop base:

$\Lambda_{\mathrm{c}}=\frac{-L}{\ln \left(\frac{\rho_{\text {apex }}}{\rho_{\text {base }}}\right)}=\frac{L}{\ln \left(\frac{\rho_{\text {base }}}{\rho_{\text {apex }}}\right)}$.

It is of interest to plot the period ratio $P_{1} / 2 P_{2}$ as a function of the density ratio $\rho=\rho_{\text {apex }} / \rho_{\text {base }}$. Figure 2 displays the results. For comparison we have also considered several other profiles: the linear profile (using the dispersion relations (29) and (31)); the profile discussed by Dymova \& Ruderman (2006a), viz.

$c_{\mathrm{k}}^{2}(z)=c_{\mathrm{k}}^{2}(0)\left[1-\left(1-\rho^{1 / 2}\right) \frac{z^{2}}{L^{2}}\right]^{2} ;$

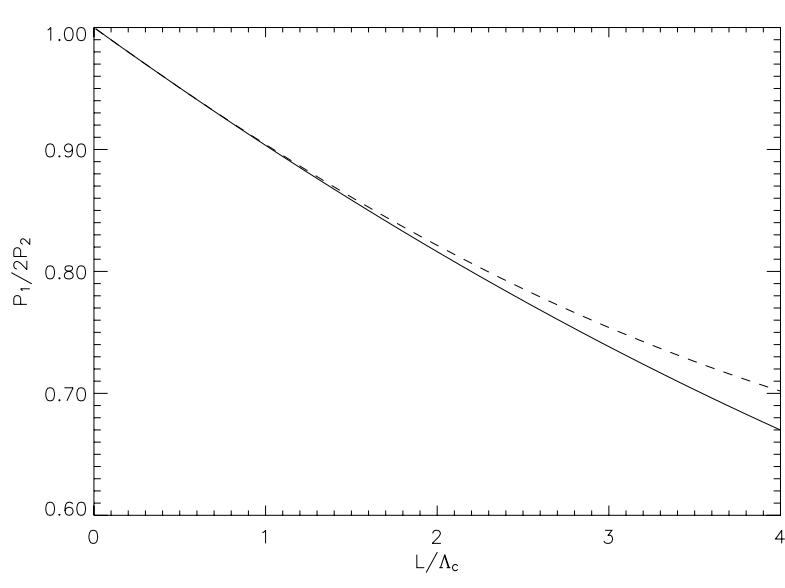

Fig. 1. The period ratio $P_{1} / 2 P_{2}$ for a thin tube with exponential longitudinal structuring. The dashed curve corresponds to the numerically determined solution of the dispersion relations (9) and (10). The solid curve corresponds to the analytical approximation Eq. (46).

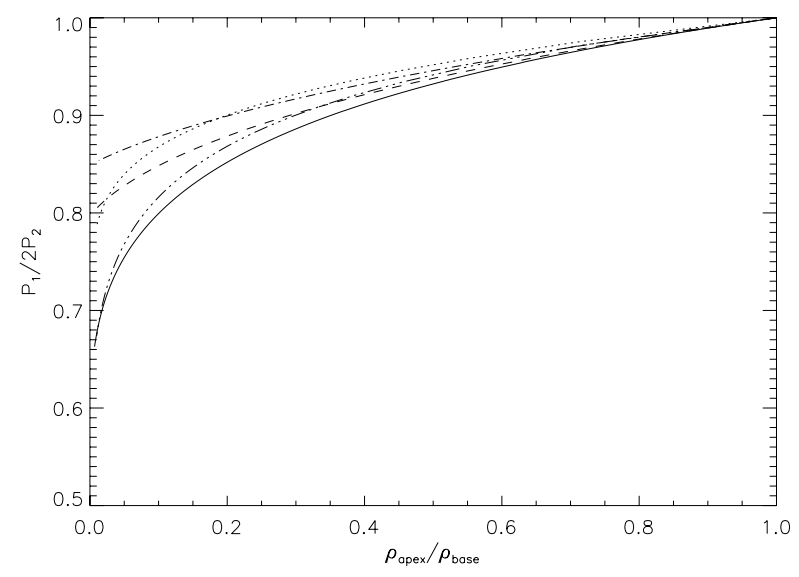

Fig. 2. The period ratio $P_{1} / 2 P_{2}$ as a function of the density ratio $\rho_{\text {apex }} / \rho_{\text {base }}$ of internal density $\rho_{\mathrm{i}}(z=0)$ at the loop apex to density $\rho_{\mathrm{i}}(L)$ at the loop base. The solid curve corresponds to the exponentially structured density profile, numerically determined by the solution of dispersion relations (9) and (10). Also shown for comparison are the results for a selection of other profiles, considering the linear profile (dashed line), the profile discussed by Dymova \& Ruderman (2006a), Eq. (48) (dotted line), the tanh profile (Eq. (50)) with $k L=1$ (dot-dashed line) and the profile discussed by Andries et al. (2005b) (Eq. (49), dot-dotdashed line); for these profiles we have used the variational formulation.

the profile discussed by Andries et al. (2005b) (after some algebraic operations to adapt it to our variables), with exponential structure and the curvature effect on the length along the loop, viz.

$c_{\mathrm{k}}^{2}(z)=c_{\mathrm{k}}^{2}(0) \rho^{1-\cos \frac{\pi z}{2 L}} ;$

and the profile

$c_{\mathrm{k}}^{2}(z)=c_{\mathrm{k}}^{2}(0)\left[\rho+(1-\rho) \frac{\tanh (k(L-z))}{\tanh (k L)}\right]$.

The tanh profile is of particular interest since it recovers the linear profile in the limit of small $k L$, but for large $k L$ (e.g. $k L=5$ or 10) it produces a boundary layer of rapid change at the base of the loop. For all of these profiles, when $\rho$ is close to unity, 
Table 1. Coronal seismology using $P_{1} / 2 P_{2}$ : scale heights.

\begin{tabular}{cccccc}
\hline \hline Case & $P_{1} / 2 P_{2}$ & Analytical $\Lambda_{\mathrm{c}}(\mathrm{Mm})$ & Numerical $\Lambda_{\mathrm{c}}(\mathrm{Mm})$ & Analytical $H_{\mathrm{c}}(\mathrm{Mm})$ & Numerical $H_{\mathrm{c}}(\mathrm{Mm})$ \\
\hline $\mathrm{C}$ & $0.91 \pm 0.04$ & $117 \pm 98$ & $121 \pm 97$ & $75 \pm 62$ & $77 \pm 62$ \\
$\mathrm{D}$ & $0.79 \pm 0.03$ & $49 \pm 9$ & $48 \pm 9$ & $31 \pm 6$ & $31 \pm 7$ \\
$\mathrm{E}$ & $0.90 \pm 0.03$ & $192 \pm 68$ & $182 \pm 68$ & $122 \pm 43$ & $116 \pm 43$ \\
\hline
\end{tabular}

Table 2. Coronal seismology using $P_{1} / 2 P_{2}$ : density ratio $\rho_{\text {apex }} / \rho_{\text {base }}$.

\begin{tabular}{ccccccc}
\hline \hline Case & $P_{1} / 2 P_{2}$ & Exponential & Linear & Eq. (48) & Eq. (49) & Eq. (50) \\
\hline $\mathrm{C}$ & $0.91 \pm 0.04$ & $0.39 \pm 0.19$ & $0.34 \pm 0.22$ & $0.25 \pm 0.24$ & $0.35 \pm 0.20$ & $0.27 \pm 0.24$ \\
$\mathrm{D}$ & $0.79 \pm 0.03$ & $0.09 \pm 0.03$ & - & $0.05 \pm 0.04$ & $0.07 \pm 0.04$ & - \\
$\mathrm{E}$ & $0.90 \pm 0.03$ & $0.35 \pm 0.11$ & $0.29 \pm 0.15$ & $0.20 \pm 0.13$ & $0.31 \pm 0.11$ & $0.21 \pm 0.16$ \\
\hline
\end{tabular}

i.e. when $\rho_{\text {apex }} \simeq \rho_{\text {base }}$, longitudinal structuring is weak and so $P_{1} / 2 P_{2}=1$, the thin tube value in a uniform medium. When longitudinal structuring is strong, so that $\rho_{\text {apex }} \ll \rho_{\text {base }}$, then $P_{1} / 2 P_{2}$ departs strongly from unity. The value of the period ratio, in the limit of $\rho_{\text {apex }} \ll \rho_{\text {base }}$, is calculated using the variational technique outlined in Sect. 2.3. For the exponential profile, using the trial functions given by Eq. (37), the period ratio $P_{1} / 2 P_{2} \simeq 0.50$ when longitudinal structuring is strong. By comparison, for the linear profile $P_{1} / 2 P_{2} \simeq 0.80$ when $\rho_{\text {apex }} \ll \rho_{\text {base }}$.

The main conclusion to be drawn from Fig. 2 is that all profiles give similar period ratios when $\rho_{\text {apex }}$ is not greatly different from $\rho_{\text {base }}$, but for $\rho_{\text {apex }}$ much smaller than $\rho_{\text {base }}$ there is a significant variation in the period ratio with choice of $c_{\mathrm{k}}^{2}(z)$ profile. Consequently, other considerations are needed before any confident deduction of $\rho_{\text {apex }} / \rho_{\text {base }}$ may be made from observational knowledge of the period ratio. Moreover, notice that different profiles give a range of values for the density ratio, once a value for $P_{1} / 2 P_{2}$ has been fixed. Therefore, it should be taken into account when using Eq. (46) that other density profiles might give different values, although it provides a handy approximation for a simple estimation of $\rho_{\text {apex }} / \rho_{\text {base }}$ which roughly agrees with similar profiles. Notice also that the profile in Eq. (49) satisfies the condition in Eq. (5), showing that the profiles which include such cusps at the apex behave similarly, and thus this cusp is a minor feature regarding the period ratio.

With this in mind, we consider the application of the results for the exponential profile to observations. Consider Eq. (46) applied to the data observed in Verwichte et al. (2004). van Doorsselaere et al. (2007) reanalysed this data obtaining a higher accuracy in determining periods and found $P_{1} / 2 P_{2}$ for two cases (labelled C and D in Verwichte et al. 2004; and van Doorsselaere et al. 2007) to be

$$
\left.\frac{P_{1}}{2 P_{2}}\right|_{\mathrm{C}}=0.91 \pm 0.04,\left.\quad \frac{P_{1}}{2 P_{2}}\right|_{\mathrm{D}}=0.79 \pm 0.03
$$

van Doorsselaere et al. (2007) also reported a new oscillation (labelled case E) that exhibited multiple harmonics, finding that

$$
\left.\frac{P_{1}}{2 P_{2}}\right|_{\mathrm{E}}=0.90 \pm 0.03
$$

These observations, of cases $\mathrm{C}, \mathrm{D}$ and $\mathrm{E}$, allows us to employ formula (46) in order to deduce the scale height $\Lambda_{c}$ of longitudinal structuring in these events.

Calculating the associated value of $L / \Lambda_{\mathrm{c}}$ from Eq. (46), for the exponential case, gives

$$
\left.\frac{L}{\Lambda_{\mathrm{c}}}\right|_{\mathrm{C}}=0.9 \pm 0.4,\left.\quad \frac{L}{\Lambda_{\mathrm{c}}}\right|_{\mathrm{D}}=2.3 \pm 0.4,\left.\quad \frac{L}{\Lambda_{\mathrm{c}}}\right|_{\mathrm{E}}=1.1 \pm 0.3
$$

Using the loop lengths of $2 L=218 \mathrm{Mm}$ for case $\mathrm{C}, 2 L=$ $228 \mathrm{Mm}$ for case D and $2 L=400 \mathrm{Mm}$ for case E we can determine the values of the density scale heights, from Eq. (46), for these three oscillating coronal loops, for the exponential profile. For case $\mathrm{C}$ we find $\Lambda_{\mathrm{c}}=117 \pm 98 \mathrm{Mm}$, for case $\mathrm{D}$ we determine $\Lambda_{c}=49 \pm 9 \mathrm{Mm}$ and for case $\mathrm{E}$ we determine $\Lambda_{\mathrm{c}}=192 \pm 68 \mathrm{Mm}$. The three cases are summarised in Table 1, where we deduce the scale $\Lambda_{c}$ of longitudinal density variation in the loops from Eq. (46) for the exponential case. We also include results from numerical solution of dispersion relations (9) and (10). Additionally, in Table 1 we have given the associated vertical scale $H_{\mathrm{c}}=2 \Lambda_{\mathrm{c}} / \pi$ which arises if the loop has a semicircular shape reaching a height of $H_{\mathrm{c}}$ at the loop apex (see also Andries et al. 2005b; and van Doorsselaere et al. 2007).

Finally, using Fig. 2 we may calculate the density ratio $\rho_{\text {apex }} / \rho_{\text {base }}$ for the three cases assuming an exponential profile. For case $\mathrm{C}$ we find $\rho_{\text {apex }} / \rho_{\text {base }}=0.41 \pm 0.20$ and for case $\mathrm{D}$ we find $\rho_{\text {apex }} / \rho_{\text {base }}=0.09 \pm 0.03$. For case $\mathrm{E}$ we determine $\rho_{\text {apex }} / \rho_{\text {base }}=0.35 \pm 0.14$. If other profiles are assumed we obtain the results in Table 2, where we deduce the density ratio $\rho_{\text {apex }} / \rho_{\text {base }}$ in the loops for different equilibrium profiles using the results in Fig. 2 (a "-" sign means that this ratio cannot be achieved with that particular equilibrium profile). These values lay in the error margin given by the uncertainty in the measurement of the periods. We conclude that in the current available observations, the choice of model is a minor source for the error in Eq. (46) compared with observational uncertainties.

\section{Conclusions}

McEwan et al. (2006) showed that the dominant cause of a shift in $P_{1} / 2 P_{2}$ from unity is the longitudinal structuring of the plasma, exceeding shifts caused by other effects such as dispersion. The observational determination of $P_{1} / 2 P_{2}$ thus yields information about the longitudinal structure. In the thin tube limit, the ratio $P_{1} / 2 P_{2}$ depends only upon the ratio $L / \Lambda_{\mathrm{c}}$ of the loop half-length $L$ to the scale height $\Lambda_{\mathrm{c}}$. It is independent of other parameters, such as the propagation speed $c_{\mathrm{k}}$. Thus by using Eq. (46) one can determine the coronal density scale height $\Lambda_{c}$ without relying on other input parameters. In the three currently known observational determinations of the periods $P_{1}$ and $P_{2}$, significant error bars arise in the determination of $\Lambda_{c}$ in each case. However, instruments and techniques for measuring periods are more advanced than those for determining number density or magnetic field strength, so we have here a method for coronal seismology that relies on relatively well known input parameters. In conclusion, the period ratio $P_{1} / 2 P_{2}$ is potentially a powerful tool for atmospheric seismology. 
Potentially, formula (46) has applications for stellar observations also. The determination of input parameters for stellar seismology is difficult, mainly due to the great distances involved. However, observations of stellar coronal oscillations have been reported (Mathioudakis et al. 2003, 2006; Mitra-Kraev et al. 2005), although only single harmonics have so far been detected. With observations of higher harmonics it should prove possible to determine stellar density scale heights.

Acknowledgements. The authors acknowledge financial support from the Particle Physics and Astronomy Research Council and thank Dr. Gavin Donnelly for useful discussions. Tom van Doorsselaere and Michael Ruderman are also acknowledged for their accurate remarks and help in the discussion of the results.

\section{References}

Abramowitz, M., \& Stegun, I. A. 1964, Handbook of Mathematical Functions (National Bureau of Standards)

Andries, J., Goossens, M., Hollweg, J. V., Arregui, I., \& Van Doorsselaere, T. 2005a, A\&A, 430, 1109

Andries, J., Arregui, I., \& Goossens, M. 2005b, ApJ, 624, L57

Bender, C. M., \& Orszag, S. A. 1978, Advanced Mathematical Methods for Scientists and Engineers (New York: McGraw-Hill)

De Moortel, I., \& Brady, C. S. 2007, ApJ, 664, 1210

Díaz, A. J. 2004, Ph.D. Thesis, Universitat de les Illes Balears

Díaz, A. J., Oliver, R., \& Ballester, J. L. 2002, ApJ, 580, 550

Díaz, A. J., Donnelly, G. R., \& Roberts, B. 2007, A\&A, 476, 359

Donnelly, G. R., Díaz, A. J., \& Roberts, B. 2006, A\&A, 457, 707
Dymova, M. V., \& Ruderman, M. S. 2005, Sol. Phys., 229, 79 Dymova, M. V., \& Ruderman, M. S. 2006a, A\&A, 457, 1059 Dymova, M. V., \& Ruderman, M. S. 2006b, A\&A, 459, 241

Dymova, M. V., \& Ruderman, M. S. 2007, A\&A, 463, 759

Edwin, P. M., \& Roberts, B. 1983, Sol. Phys., 88, 179

Erdélyi, R., \& Verth, G. 2007, A\&A, 462, 743

Ferriz Mas, A., \& Schüssler, M. 1989, Geophys. Astrophys. Fluid Dyn., 48, 217 Goossens, M., Andries, J., \& Arregui, I. 2006, Roy. Soc. Lond. Phil. Trans. Ser. A, 364, 433

Mathioudakis, M., Bloomfield, D. S., Jess, D. B., Dhillon, V. S., \& Marsh, T. R. 2006, A\&A, 456, 323

Mathioudakis, M., Seiradakis, J. H., Williams, D. R., et al. 2003, A\&A, 403, 1101

McEwan, M. P., Donnelly, G. R., Díaz, A. J., \& Roberts, B. 2006, A\&A, 460, 893

Mitra-Kraev, U., Harra, L. K., Williams, D. R., \& Kraev, E. 2005, A\&A, 436, 1041

Morse, P. M., \& Feshbach, H. 1953, Methods of theoretical physics, International Series in Pure and Applied Physics (New York: McGraw-Hill)

Nakariakov, V. M., \& Ofman, L. 2001, A\&A, 372, L53

Nakariakov, V. M., \& Verwichte, E. 2005, Living Rev. Sol. Phys.,

http://solarphysics. livingreviews.org/lrsp-2005-3

Roberts, B., Edwin, P. M., \& Benz, A. O. 1984, ApJ, 279, 857

Roberts, B., \& Webb, A. R. 1978, Sol. Phys., 56, 5

Roberts, B., \& Webb, A. R. 1979, Sol. Phys., 64, 77

Ryutov, D. D., \& Ryutova, M. P. 1976, Sov. Phys, 43, 491

Spruit, H. C. 1981, A\&A, 102, 129

Stix, M. 2004, A\&A, 415, 751

van Doorsselaere, T., Nakariakov, V. M., \& Verwichte, E. 2007, A\&A, 473, 959

Verwichte, E., Nakariakov, V. M., Ofman, L., \& Deluca, E. E. 2004, Sol. Phys., 233, 77 